\title{
Scurvy: A Case Report on a Child With Autism
}

\author{
Pei Pei Lima, c, Bahadun Juanna ${ }^{\mathrm{b}}$
}

\begin{abstract}
Scurvy is a nutritional deficiency which has currently become uncommon. Accurate history taking, strong suspicion and good clinical correlation especially in high risk groups are important in making an early diagnosis. A 9-year-old boy with underlying autism was presented to the Pediatric Dental Clinic with gingival swelling, fever and refusal to walk. The child was subsequently referred to the Pediatric Medical team for suspicion of a hematological malignancy. After a thorough assessment, he was diagnosed of having scurvy due to his peculiar diet habit. Vitamin $\mathrm{C}$ supplementation was started as diagnostic and therapeutic measures. Scurvy is a disease caused by chronic vitamin $\mathrm{C}$ deficiency. Though it has become uncommon, it still exists in high risk populations. The clinical manifestation of scurvy could be variable and non-specific. In practice, the diagnosis of scurvy is based on history and clinical findings. In cases of suspicion, resolution of disease manifestations after vitamin $\mathrm{C}$ supplementation remains the best diagnostic evidence. High index of suspicion is crucial to diagnose scurvy early in modern era. Early diagnosis is important for prompt initiation of treatment and fast resolution of symptoms.
\end{abstract}

Keywords: Scurvy; Vitamin C; Ascorbic acid; Autism

\section{Introduction}

Scurvy is a disease caused by chronic vitamin C deficiency, which is an essential micronutrient to the human body [1]. Humans are unable to convert glucose to vitamin $\mathrm{C}$ via l-gulonolactone oxidase $[1,2]$. Hence, the source of vitamin $\mathrm{C}$ depends exclusively from dietary intake. Vitamin $\mathrm{C}$ is essential in formation of intercellular connective tissue, osteoid, dentine and collagen. Lacking in Vitamin $\mathrm{C}$ will lead to a vast spectrum of clinical manifestations due to its role in various biological

Manuscript submitted November 19, 2018, accepted November 29, 2018

aDepartment of Pediatric, Hospital Shah Alam, Seksyen 7, Persiaran Kayangan, 40000 Shah Alam, Malaysia

bepartment of Pediatric Dentistry, Hospital Shah Alam, Seksyen 7, Persiaran Kayangan, 40000 Shah Alam, Malaysia

${ }^{\mathrm{c} C}$ Corresponding Author: Pei Pei Lim, Department of Pediatric, Hospital Shah Alam, Seksyen 7, Persiaran Kayangan, 40000 Shah Alam, Malaysia.

Email: limpeipei@moh.gov.my

doi: https://doi.org/10.14740/ijcp321 processes.

Scurvy has been reported as ancient as dated to Egyptian age. A skeleton of an infant from the ancient Egyptian settlement of Nag el-Qarmila (c. 3800 - 3600 B.C.E.) may provide the earliest attested evidence of scurvy [3]. It used to be a disease with high mortality as United East India Company (VOC) lost more sailors on scurvy than on the battlefield during the 17 th century [4]. The first ever recorded clinical trial was conducted by Sir James Lind associating prevention of scurvy and taking citrus fruits in $1747[5,6]$.

Over years, as the food sources become easily available and healthcare service has improved tremendously, it was thought that scurvy, being one of the most ancient nutritional deficiencies in the world, should have been extinct.

\section{Reemerging of vitamin C deficiency}

Despite of long thought to be extinct, scurvy still present in the modern age even in the most developed regions. Certain populations who are vulnerable to nutritional deficiency due to their underlying co-morbidity are particularly at risk. These include people with specific dietary habits, alcoholic or institutionalized [5]. Children with neurodevelopmental and behavioral disorders such as cerebral palsy or autism are susceptible due to their dietary restrictions [7]. Because of its rarity, it is often overlooked and patients were often subjected to unnecessary investigations which further delaying the diagnosis.

We report a case of an autistic child who presented with gingival swelling and refusal to walk. The initial impression was hematological malignancy before the diagnosis of scurvy was considered.

\section{Case Report}

An 8-year-7-month old autistic boy was referred to the Department of Pediatric Dentistry for gum swelling associated with bleeding for 2 weeks. History was fully taken from the mother as child was non-verbal. Three weeks prior, he had high-grade fever with chills and rigor. Fever lasted for 2 weeks, during which the gum swelling developed.

The child had poor appetite and significant weight loss. He had lost $7 \mathrm{~kg}$ in 3 weeks' time. A week earlier, the child had a fall and fracture was ruled out by bilateral knee radiographs. However, since then mother noticed he had been having difficulty walking. His condition worsened to the extent of needing 

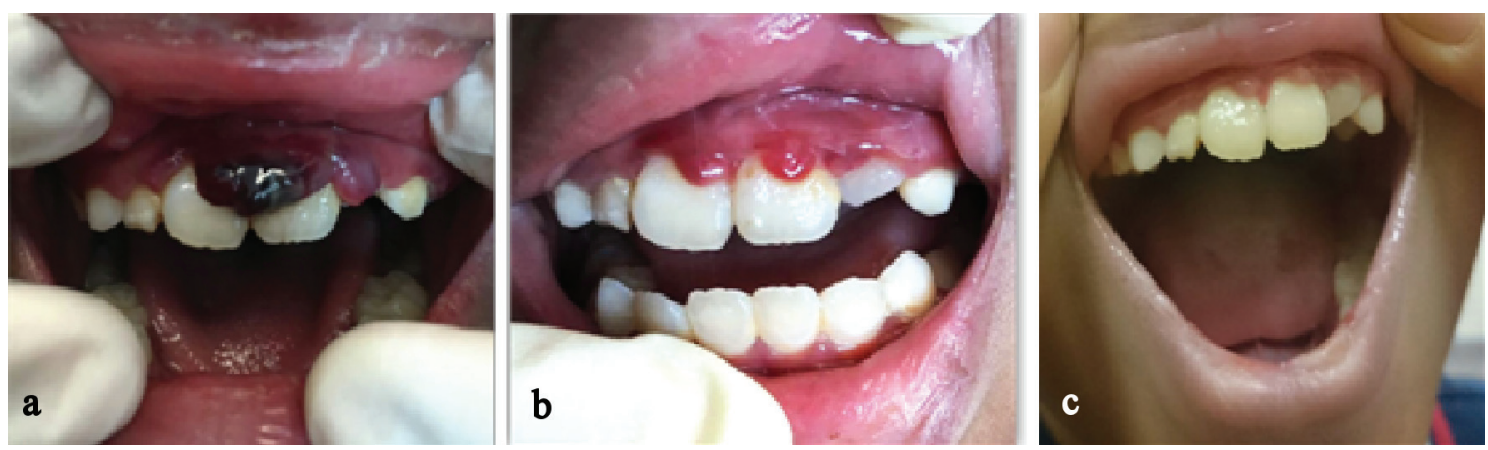

Figure 1. (a) Gum hypertrophy prior to vitamin C treatment. (b) Gum hypertrophy on day 5 of vitamin C treatment. (c) Gum hypertrophy resolving 1 month post vitamin $\mathrm{C}$ treatment.

his mother to assist him in rising up from sitting position.

He was diagnosed to have autism at 4 years old but was not on regular follow-up. He was born of non-consanguineous marriage. His mother had South East Asia ovalocytosis but was asymptomatic. He was the youngest of four siblings and his elder brother was a thalassemia carrier. His other immediate family members were healthy.

During clinic review, the child looked restless and miserable. Intra-orally, there were several areas of hypertrophic and erythematous gingiva particularly in the labial and palatal gingiva of maxillary anterior teeth, lingual gingiva of mandibular anterior teeth and buccal gingiva of mandibular posterior teeth (Fig. 1a). The gums were red and purplish in color and bled easily upon contact. The swelling were almost symmetrical in locations. Plaque was minimal and unlikely to cause such swelling. There were no significant extra-oral findings and lymph nodes were non-palpable. His liver and spleen were not enlarged. In view of the gum swelling with constitutional symptoms, hematological malignancy was highly suspected.

The child was febrile and admitted to the Pediatric Ward. In ward, he was found to have multiple petechiae on bilateral shins which were not blanchable (Fig. 2a). There was no musculoskeletal deformity. No neurological deficit was found on examination.

The laboratory workup was not remarkable for malignancy (white blood cell count: 10,220/ $\mu \mathrm{L}$, platelets count: $440,000 /$ $\mu \mathrm{L}$, hemoglobin: $10.5 \mathrm{~g} / \mathrm{dL}$ (microcytic hypochromic anemia), blood sedimentation rate $>140 \mathrm{~mm} / \mathrm{h}$ and a C-reactive protein of $90 \mathrm{mg} / \mathrm{dL}$ ). Coagulation profile, alkaline phosphatase, calcium and phosphate levels were all normal. The peripheral blood film did not show abnormal cells. Radiograph of both his lower limbs were unremarkable.

His fever was persistently high with body temperature between $39{ }^{\circ} \mathrm{C}$ to $40.5{ }^{\circ} \mathrm{C}$. Third generation cephalosporin was empirically started but no clinical improvement. In view of high suspicion of malignancy, oral and bone marrow biopsies were contemplated.

Before the decision of biopsy was made, we noticed the child had a peculiar dietary habit in ward. He refused all food being served in hospital and would only consume the food brought from home, namely instant noodles, rice, plain soup and biscuits. Further questioning revealed the fact that he had been consuming the same instant noodles without any added ingredients or vegetables for the past 6 month. In between meals, he would snack on his favorite biscuits which contain no vitamin $\mathrm{C}$. He used to take chewable vitamin $\mathrm{C}$ daily but has stopped it since his parents separated 6 months earlier. His food aversion raised the suspicion of scurvy.

In view that measurement of vitamin $\mathrm{C}$ level was not available in our facility; oral vitamin $\mathrm{C}$ was commenced as both diagnostic and therapeutic measures. He was given 100

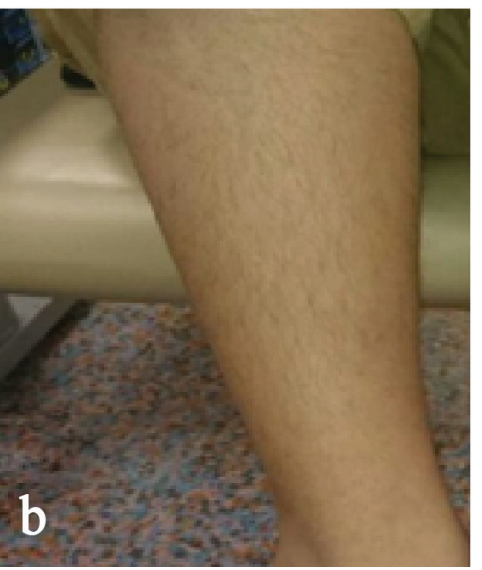

Figure 2. (a) Petechiae on shin prior to vitamin $C$ treatment. (b) Petechiae fully resolved 1 month after vitamin $C$ treatment. 
$\mathrm{mg}$ vitamin $\mathrm{C}$ three times a day.

\section{Progress of the disease}

The fever subsided within $24 \mathrm{~h}$ after the commencement of vitamin C. Few days later, the gingival hypertrophy showed significant improvement. The swelling reduced and became less reddish. The petechiae on the shins became less and the fever resolved within 1 week. Patient became more cheerful. He was discharged 1 week after with oral vitamin C $100 \mathrm{mg}$ three times a day. He began ambulating 1 week after discharge. At 1-month review, he was fully recovered and back to his normal cheerful self, his weight increased from $21 \mathrm{~kg}$ to $24.7 \mathrm{~kg}$ in 1 month. He was able to ambulate and run as usual.

\section{Discussion}

At present day, it is generally believed that dietary excess is more commonly seen in most of the regions compared to nutritional deficiency. However, nutritional deficiency such as scurvy still occurs even in the most developed regions. In adults, it occurs in uncared elderly who are living alone, alcoholics, food faddists, and those who are suffering for psychiatric disorders $[1,5]$. In pediatric population, those who are susceptible include children with dietary restrictions due to neuropsychiatric or developmental disorders $[1,7,8]$. Children with autism tend to demonstrate highly restrictive behavior which may significantly limits their ability to accept food with varied tastes, textures or even colors. As a result, they usually follow an incredibly rigid menu for long period of time. This will definitely put them at high risk for nutritional deficiency conditions, including scurvy.

Vitamin $\mathrm{C}$ is a water-soluble vitamin, with a half-life of 10 to 20 days [8]. However, signs of deficiency starts manifest after 1 to 3 months of insufficient vitamin C intake [8-10]. The earliest manifestations of scurvy are nonspecific constitutional symptoms, such as lethargy, anorexia, and weight loss [9]. As the condition progress, patient may present with gingival hyperplasia and bleeding in the skin and joints. This is due to poorly formed collagen causing weakened blood vessel wall. In our patient, he had been taking restricted diet about 6 months prior to the symptoms. He demonstrated significant constitutional symptoms especially fever and weight loss due to reduced appetite. This followed by gum swelling and follicular hemorrhage which gave rise to the petechial lesions. His refusal to walk was largely attributed to bone pain. Due to its multifaceted clinical manifestations, children with scurvy may present themselves to a variety of disciplines ranging from Pediatrics, Dentistry, Orthopedic, Rheumatology, Neurology and even Oncology. Hence, high index of suspicion is important to clinch the diagnosis.

Diagnosis of scurvy could be made clinically with a meticulous clinical evaluation, including a comprehensive history taking and physical examination [8]. Laboratory workup is not compulsory to confirm the diagnosis $[8,11]$. Laboratory test abnormalities in scurvy are nonspecific; anemia is a frequent manifestation which could be multifactorial. It could be secondary to bleeding and iron deficiency as vitamin $\mathrm{C}$ deficiency itself could lead to decrease in iron absorption [12-13]. In this patient, we did not pursue serum vitamin $\mathrm{C}$ level as it was not available in local healthcare facility. Moreover, outsourcing will be costly and its turn-around time is long. Thirdly, we do not need the result before initiating treatment as vitamin $\mathrm{C}$ is safe and easy to administer and clinical improvement could be anticipated within a short period of time. Overall improvement of symptoms after the administration of ascorbic acid is a confirmatory approach for the diagnosis [14].

Treatment of scurvy is based on replenishing the level of vitamin $\mathrm{C}$ to counteract the symptoms. There is no established regimen for vitamin $\mathrm{C}$ supplementation in scurvy [8]. A recommended dose of $200 \mathrm{mg}$ per day results in marked improvement within several days [9]. Other recommendations are that 1 to $2 \mathrm{~g}$ of vitamin $\mathrm{C}$ daily for the first 2 to 3 days followed by $500 \mathrm{mg}$ per day for the next week [10]. Afterward, a daily intake of $100 \mathrm{mg}$ of vitamin $\mathrm{C}$ should be given for 1 to 3 months [10]. Another recommended dosing for children is $100 \mathrm{mg} 3$ times a day for 1 week and is then decreased to once daily, with complete recovery expected in several months [11]. Once vitamin $\mathrm{C}$ deficiency has been treated, prevention and correction of underlying conditions are required in order to prevent recurrence. We have decided to start our patient on $100 \mathrm{mg}$ three times a day coincided with his meal time. After his symptoms had fully resolved, we reduced the dose to $100 \mathrm{mg}$ daily which was the usual maintenance dose to prevent scurvy [15]. Apart from vitamin $\mathrm{C}$, the patient was also given multivitamin as we anticipated other concurrent nutritional deficiency.

Following treatment with vitamin $\mathrm{C}$, it is anticipated that symptoms of fatigue, lethargy, pain, anorexia, and confusion should improve within $24 \mathrm{~h}$ of supplementation [10], while bruising, perifollicular hemorrhages, gingival bleeding, and weakness usually improve within 1 to 2 weeks [10]. In our patient, he showed improvement within $24 \mathrm{~h}$ when his fever subsided completely. His gingival hypertrophy showed significant improvement within 5 days (Fig. 1b) and resolved after 1 month (Fig. 1c). The petechiae on the shins resolved within 1 week (Fig. 2b). Within 1 month, he was able to ambulate and run as usual.

\section{Conclusions}

Scurvy is an uncommon disease nowadays but it still exists especially in certain susceptible groups. High index of suspicion together with detailed history taking are important to diagnose it early which lead to prompt initiation of treatment. Initiation of vitamin $\mathrm{C}$ could be both as diagnostic and therapeutic measures. Management for scurvy is easy and cheap but it could be fatal if not been recognized and treated in time.

\section{Acknowledgments}

We thank the DG of health, Ministry of Health, Malaysia for permission to publish this report. We also would like to ex- 
press our gratitude to everybody who involved in this study.

\section{Conflict of Interest}

None.

\section{References}

1. Agarwal A, Shaharyar A, Kumar A, Bhat MS, Mishra M. Scurvy in pediatric age group - A disease often forgotten? J Clin Orthop Trauma. 2015;6(2):101-107.

2. Figueroa-Mendez R, Rivas-Arancibia S. Vitamin C in health and disease: its role in the metabolism of cells and redox state in the brain. Front Physiol. 2015;6:397.

3. Pitre MC, Stark RJ, Gatto MC. First probable case of scurvy in ancient Egypt at Nag el-Qarmila, Aswan. Int J Paleopathol. 2016;13:11-19.

4. Wijkmans RA, Talsma K. Modern scurvy. J Surg Case Rep. 2016;2016(1):rjv168.

5. Francescone MA, Levitt J. Scurvy masquerading as leukocytoclastic vasculitis: a case report and review of the literature. Cutis. 2005;76(4):261-266.

6. Weinstein M, Babyn P, Zlotkin S. An orange a day keeps the doctor away: scurvy in the year 2000. Pediatrics.
2001;108(3):E55.

7. Valentini D, Barbuti D, Grandin A, De Horatio LT, Villani A. A good growth in a child with scurvy. BMJ Case Rep. 2011;2011.

8. Alqanatish JT, Alqahtani F, Alsewairi WM, Al-kenaizan S. Childhood scurvy: an unusual cause of refusal to walk in a child. Pediatr Rheumatol Online J. 2015;13:23.

9. Allgaier RL, Vallabh K, Lahri S. Scurvy: A difficult diagnosis with a simple cure. African Journal of Emergency Medicine. 2012;2:20-23.

10. Leger D. Scurvy: reemergence of nutritional deficiencies. Can Fam Physician. 2008;54(10):1403-1406.

11. Meghan Cain, et al. Ascorbic acid deficiency (Scurvy) in a Toddler with restricted dietary intake presenting with "Leg Weakness" and a rash. ICAN Infant Child \& Adolescent Nutrition. 2014;6(4):201-204.

12. Desai VD, Hegde S, Bailoor DN, Patil N. Scurvy extinct? Think again! Int J Clin Pediatr Dent. 2009;2(3):39-42.

13. Velandia B, Centor RM, McConnell V, Shah M. Scurvy is still present in developed countries. J Gen Intern Med. 2008;23(8):1281-1284.

14. Baradhi KM, Vallabhaneni S, Koya S. Scurvy in 2017 in the USA. Proc (Bayl Univ Med Cent). 2018;31(2):227228.

15. Frei B, Trabe MG. The new US Dietary Reference Intakes for vitamins C and E. Redox Rep. 2001;6(1):5-9. 